\title{
Numerical modeling of surface treatment by laser beam
}

\author{
R. Gospavic \& V. Popov \\ Wessex Institute of Technology, Ashurst Lodge, Ashurst, Southampton, \\ $U K$
}

\begin{abstract}
In this work an analytical approach for analysis of surface treatment by a laser beam is presented. A thermal model of interaction for the case of cylindrical geometry of the material and asymmetric distribution of the laser beam intensity are used. An analytical procedure is developed to analyze the temporal and the spatial distribution of the temperature field inside the bulk of material. This kind of consideration is of practical interest in cases where the excitation by the laser beam is not symmetric in respect to its position or shape, e.g. multi-mode working regimes or asymmetrical distribution of the laser beam intensity. The heating effects were considered in the temperature range up to the melting point. The thermal and the optical parameters of the material were assumed to be independent of the temperature and were given constant values in the temperature range of interest. This approach makes use of the Laplace transform, in order to eliminate dependence on time. The Fourier method of variable separation was used to obtain the temperature field distribution in the Laplace transform domain.
\end{abstract}

Keywords: surface treatment, laser, thermal model, multi-mode.

\section{Introduction}

In a general case the analysis of the laser-material interaction, important for practical applications, is very complex and includes analysis of different physical processes such as material removal, material melting, thermal stresses, shock wave, etc. This prevents successful construction of a general analytical solution and different numerical procedures have been used in the past [1]. This work is restricted only to analysis of heating effects of the laser-material interaction. 
In many practical applications of laser beams the finite dimensions of the bulk material and the asymmetric distribution of the laser beam intensity has to be taken into account. The presented analytical approach enables consideration of the heating effects of interactions for the different and complicated laser-beam distributions for cases without axial symmetry. The temperature field distribution in this way can be analyzed for 3D cylindrical geometry allowing monitoring of the time evolution of the temperature distribution. For the arbitrary time dependence, spatial distribution and position of the incoming laser beam intensity the same numerical data could be used, saving in this way memory and CPU time. By using Duhamel's principle [2] the temperature distribution is evaluated by using the convolution integral.

This kind of analyses could be important in many technical applications of laser beams in technology and science [3-5] as well as in the case of lasermaterial interaction in the multi-mode working regime.

For the multi-mode working regime as well as for the complex laser beam intensity distribution superposition principle could be used. In case of the linear governing partial differential equation (PDE) the final solution could be presented as superposition or sum of the PDE solutions belonging to different parts of the incident loads, i.e. in our cases the incoming laser beam intensity.

\section{Mathematical model}

Heating of a homogenous cylinder, with a finite or infinite length, by an incident laser beam on the upper surface of the specimen is considered (Fig. 1). The shape of the cross-section, the position of the laser beam on the upper side of the specimen, the distribution and the time dependence of the laser beam intensity can be arbitrary. In the numerical examples presented in this paper, because of simplicity and still without losing generality, only a top head laser beam profile with circular cross section is considered. It was assumed that the laser beam intensity could be approximated by a product of two functions of spatial and time coordinates: $q(r)$ and $\varphi(t)$, respectively $[5,8]$.

Only the heating effects due to the interaction were considered. The lasermaterial interaction was modeled by the equivalent thermal flux on the upper side of the specimen. All thermal and optical parameters of the material are considered to be constant and temperature independent, yielding a linear thermal conduction problem. The geometry of the considered problem was represented in a cylindrical reference system.

The temperature distribution inside the bulk material was considered and the convective thermal losses from the lower and the axial surface of the material were taken into account, while the thermal losses from the upper surface of the specimen are neglected. The radiative losses have important contribution to the whole thermal losses at the very high temperatures [8]. Thus, for low temperature of the specimen, the radiative heat losses are smaller than convective ones and could be neglected [8]. Beside this, if the absorption length, for considered laser beam and material, is very short, related to size of the heating affected zone (HAZ), it could be considered that laser beam is absorbed by the 
surface of the specimen. This is the case for many materials of interest, thus the laser-material interaction could be approximated by the equivalent surface thermal source [8]. Constant and temperature independent value of the coefficient of thermal conductivity were assumed.

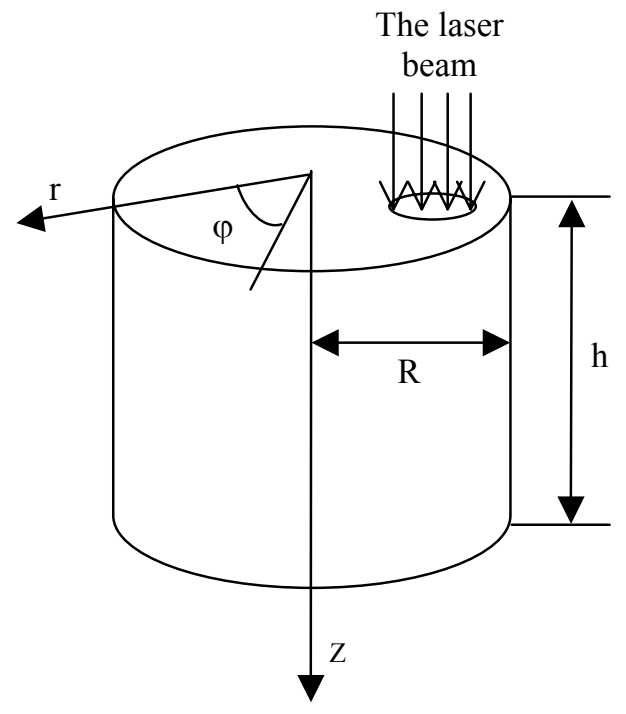

Figure 1: The geometry of considered problem and coordinate system used.

Once these approximations are applied, the heating of the considered cylindrical specimen can be modeled by the following PDE and the corresponding boundary (BC) and initial (IC) conditions [5, 9]:

$$
\begin{gathered}
\left.\frac{\partial^{2} T}{\partial r^{2}}+\frac{1}{r} \frac{\partial T}{\partial r}+\frac{\partial^{2} T}{\partial z^{2}}+\frac{\partial^{2} T}{\partial \theta^{2}}=\frac{1}{\alpha} \frac{\partial T}{\partial t} ; t \geq 0 ; 0 \leq r \leq R ; 0 \leq z \leq H ; 0 \leq \theta \leq 2 \pi \text { (1a }\right) \\
-\left.\lambda \frac{\partial T(r, z, \theta, t)}{\partial z}\right|_{z=0}=A q(r, \theta) \phi(t) ; \quad-\left.\lambda \frac{\partial T(r, z, \theta, t)}{\partial r}\right|_{r=R}=\left.h_{c} \cdot T(r, z, \theta, t)\right|_{r=R} \\
-\left.\lambda \frac{\partial T(r, z, \theta, t)}{\partial z}\right|_{z=H}=\left.h_{c} \cdot T(r, z, \theta, t)\right|_{z=H} \\
T(r, z, \theta, t=0)=0 ; \quad 0 \leq r \leq R, \quad 0 \leq z \leq H, \quad 0 \leq \theta \leq 2 \pi
\end{gathered}
$$

where: $\lambda$ is the coefficient of thermal conductivity, which is considered to be constant and temperature independent, $\alpha=\lambda / \rho \cdot c$ is the thermal diffusivity, $c$ is the specific heath, $\rho$ is the material density, $h_{\mathrm{c}}$ is the heath transfer coefficient [10], $A$ is the absorption coefficient of the laser radiation by the material [11], $R$, $h$ are the radius and length of the specimen, respectively, and $T$ is the 
temperature difference in the interior domain relative to the ambient one. The equation (1b) represents the homogenous BCs while the equation (1c) describes the IC.

The Laplace transform approach was used to eliminate the time dependence and to convert the original problem to the equivalent problem in the Laplace transform domain [12]. Fourier's method of variable separation was used in order to transform the original PDE into three ordinary differential equations as follows [12]:

$$
\begin{aligned}
& r^{2} \frac{\partial^{2} T_{r}(r)}{\partial r^{2}}+r \frac{\partial T_{r}(r)}{\partial r}+\left(\mu^{2} \cdot r^{2}-m^{2}\right) T_{r}(r)=0 \\
& \frac{\partial^{2} T_{z}^{*}(z, s)}{\partial z^{2}}-\left(\mu^{2}+\frac{s}{\alpha}\right) \cdot T_{z}^{*}(z, s)=0 \\
& \frac{\partial^{2} T_{\theta}(\theta)}{\partial \theta^{2}}+m^{2} T_{\theta}(\theta)=0 ; \quad T^{*}(r, z, \theta, s)=T_{r}(r) \cdot T_{\theta}(\theta) \cdot T_{z}^{*}(z, s)
\end{aligned}
$$

where $\mu$ and $m$ are constants, $s$ is a complex parameter, and the asterisk in the superscript denotes functions in Laplace's transform domain. The particular solutions of the governing PDE can be expressed in the following form in the Laplace transform domain $[9,13,14]$ :

$$
\begin{aligned}
& T_{m n}^{*}(r, z, \theta, s)=T_{r}(r) \cdot T_{\theta}(\theta) \cdot T_{z m n}^{*}(z, s) ; \\
& T_{r}(r)=J_{m}\left(\mu_{m n} \cdot r\right) ; \quad T_{\theta}(\theta)=K_{1 m} \cos (m \cdot \theta)+K_{2 m} \sin (m \cdot \theta) ; \quad n=1,2, \ldots
\end{aligned}
$$

where $m$ is integer, $T(\theta)=T(\theta+2 m \pi)$, because of the continuity condition, $J_{m}$ are Bessel functions of the m-th kind, $\mu_{m n}$ are positive roots of the characteristic transcendent equations which describe the $\mathrm{BC}$ on the axial boundary surface of the specimen, given by the next relation:

$$
-\lambda \frac{\mu_{m n}}{2}\left(J_{m+1}\left(\mu_{m n} R\right)-J_{m-1}\left(\mu_{m n} R\right)\right)=h_{c} \cdot J_{m}\left(\mu_{m n} R\right)
$$

The next relations hold: $\mu_{m,-n}=-\mu_{m n},\left(\mu_{m n} \geq 0\right)$ and $T_{r m-n}^{*}=(-1)^{-m} T_{r m n}^{*}$. Due to the boundary conditions on the lower surface of the specimen and according to previous work [9], $T_{z m n}^{*}(z, s)$ could be expressed in the following way:

$$
T_{z m n}^{*}(z, s)=T_{0 m n}^{*}(s) \cdot\left(\exp \left(\frac{i z \cdot \varepsilon}{h}\right)+\frac{i \lambda \cdot \varepsilon / h+h_{c}}{i \lambda \cdot \varepsilon / h-h_{c}} \exp \left(i \frac{2 \cdot h-z}{h} \varepsilon\right)\right) ; \quad i \varepsilon=h \sqrt{\mu_{m n}^{2}+\frac{s}{\alpha}} .
$$


If the laser beam have rounded cross-section and top head profile, because of symmetry the particular solutions given by (2) have to be odd functions of the angular coordinate $\theta$ and accordingly $K_{1 m}$ and $K_{2 m}$ in (3) can be $K_{1 m}=1$ and $K_{2 m}=0$.

As the particular solutions, for positive values of the constants $\mu_{m n}$, are linearly independent, the solution in the Laplace transform domain could be evaluated by the series of the particular ones:

$$
T^{*}(r, z, \theta, s)=\sum_{n=1}^{+\infty} \sum_{m=0}^{+\infty} a_{m n} \cdot T_{r m n}(r) \cdot T_{\theta m}(\theta) \cdot T_{z m n}^{*}(z, s)
$$

The response to Dirac's pulse induction in time domain $T_{\delta}(r, z, \theta, t)$ was obtained using inverse Laplace's transformation and Bromwich integral, and it can be expressed in the following form:

$$
\begin{aligned}
& T_{\delta}(r, z, \theta, t)=L^{-1}\left\{T^{*}(r, z, \theta, s)\right\}=\int_{p-i \infty}^{p+\infty} T^{*}(r, z, \theta, s) \cdot \exp (s t) \cdot d s \Rightarrow \\
& T_{\delta}(r, z, \theta, t)=\frac{2 \cdot \alpha \cdot A}{h \lambda} \sum_{m, n, j=1}^{N} J_{m}\left(\mu_{m n} r\right) E_{j}(z) \cdot\left(c_{1 m n} \cos (m \theta)+c_{2 m n} \sin (m \theta)\right) \exp \left(\frac{-t}{\tau_{m n j}}\right)
\end{aligned}
$$

where $\mathrm{p}$ is the positive real parameter; $L^{-1}$ denotes the inverse Laplace-s transformation; $\tau_{m n j}$ and $E_{j}(z)$ are given by next relations:

$$
\tau_{m n j}=\frac{1}{\alpha\left(\mu_{m n}^{2}+\left(\frac{\varepsilon_{j}}{h}\right)^{2}\right)} ; \quad E_{j}(z)=\frac{\lambda \varepsilon_{j} \cos \varepsilon_{j}(1-z / h)+h_{c} \cdot h \sin \varepsilon_{j}(1-z / h)}{\left(\lambda+h_{c} \cdot h\right) \sin \varepsilon_{j}+\lambda \varepsilon_{j} \cos \varepsilon_{j}}
$$

For arbitrary time dependence of the laser beam intensity, the temperature distribution inside the specimen could be evaluated by a convolution integral as $[9,12]$ :

$$
T(r, z, \theta, t)=\int_{0}^{t} \phi(t-\tau) \cdot T_{\delta}(r, z, \theta, \tau) \cdot d \tau
$$

\section{Numerical examples}

In this section according to the above considerations numerical examples are presented for some characteristic cases. A cylindrical Al specimen is considered 
and the following characteristic parameters were assumed: $\lambda=240[\mathrm{~W} / \mathrm{K} \cdot \mathrm{m}]$; $\rho=2700\left[\mathrm{~kg} / \mathrm{m}^{3}\right] ; \mathrm{c}=1021.71[\mathrm{~J} / \mathrm{kg} \mathrm{K}] ; h_{\mathrm{c}}=10\left[\mathrm{~W} / \mathrm{Km}^{2}\right] ; A=0.64 ; h=5[\mathrm{~mm}] ;$ $R=7[\mathrm{~mm}]$.

In Figs. 2 the contour lines for the temperature field on the upper surface $(\mathrm{X}-$ $\mathrm{Y}$ plane) of the specimen are presented. The laser beam with top head profile was assumed to have the following characteristics: power $=500 \mathrm{~W}$, radius $=$ $1 \mathrm{~mm}$, time duration $=1 \mathrm{~s}$, coordinates of the laser beam center: $\theta_{0}=0 ; r_{1}=4 \mathrm{~mm}$.

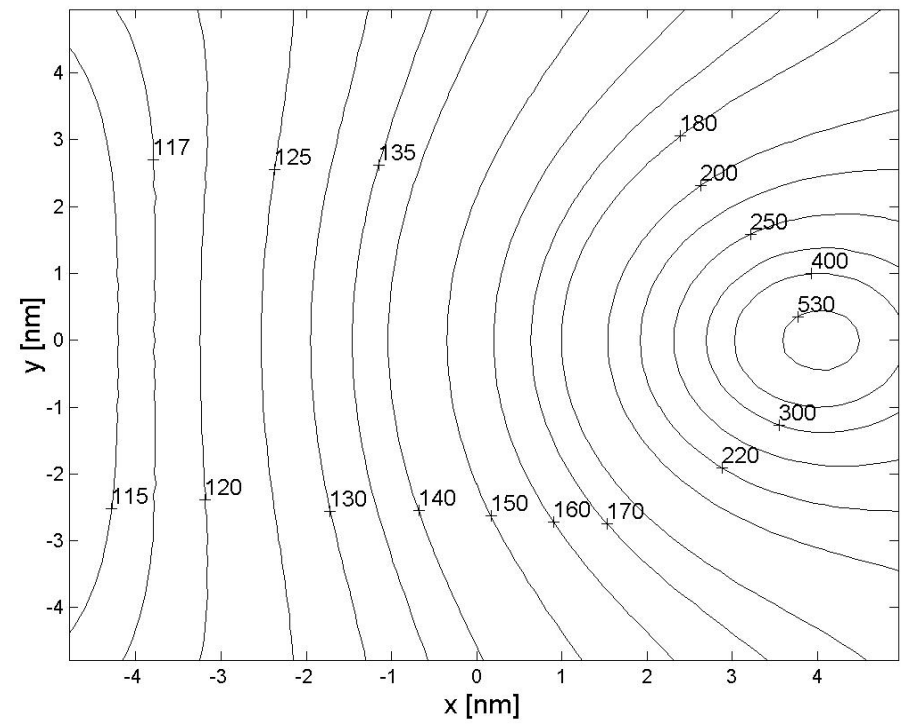

Figure 2: Contour plot of temperature on the upper surface of the specimen. The laser beam properties were: power $=500 \mathrm{~W}$, radius $=1 \mathrm{~mm}$, time duration $=1 \mathrm{~s}$, position $\theta_{0}=0 ; r_{1}=4 \mathrm{~mm}$.

In Fig. 3 the contour plot of the temperature field in $\mathrm{x}-\mathrm{z}$ plane is presented. The laser beam and the specimen parameters are the same as in the previous case.

In Fig. 4 the contour plot of the temperature difference on the upper surface ( $\mathrm{x}-\mathrm{y}$ plane) is presented, for the case of two laser beams with the same top head profiles. The laser beams have the following properties: power $P_{1}=500 \mathrm{~W} ; P_{2}=500 \mathrm{~W} ; \quad$ radii: $r_{01}=r_{02}=0.5 \mathrm{~mm} ;$ time duration $1 \mathrm{~s}$, positions of the laser beams center: $r_{1}=2 \mathrm{~mm} ; \theta_{01}=0 \mathrm{rad} ; r_{2}=3.2 \mathrm{~mm} ; \theta_{02}=0$ rad.

In Fig. 5 the contour plot in the $\mathrm{x}-\mathrm{Z}$ plane, for the same case, was presented. The dimensions and assumed physical properties of the specimen were the same as in the previous cases. 




Figure 3: Contour plot in the $\mathrm{x}-\mathrm{z}$ plane, laser beam properties: power $=500 \mathrm{~W}$, radius $=1 \mathrm{~mm}$, time duration $=1 \mathrm{~s}$, position $\theta_{0}=0 ; r_{1}=4 \mathrm{~mm}$.

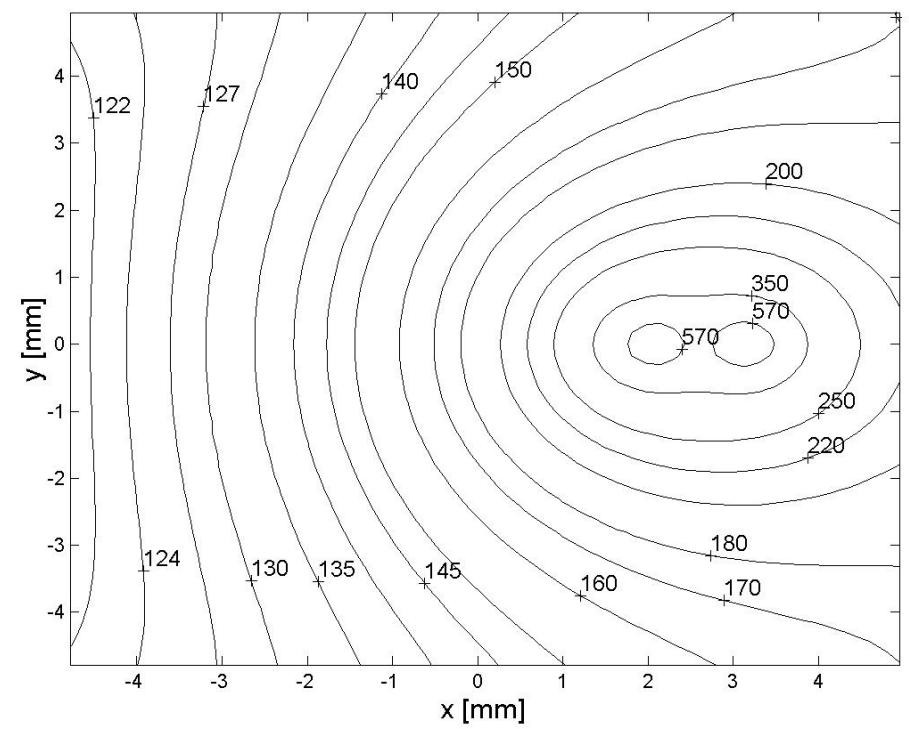

Figure 4: Contour plot of temperature on the upper surface of the specimen, for two incident laser beams, positions of the laser beams: $r_{1}=$ $2 \mathrm{~mm} ; \theta_{01}=0 \mathrm{rad} ; r_{2}=3.2 \mathrm{~mm} ; \theta_{02}=0 \mathrm{rad}$. 




Figure 5: Contour plot in the $\mathrm{x}-\mathrm{z}$ plane, for two incident laser beams, positions of the laser beams: $r_{1}=2 \mathrm{~mm} ; \theta_{01}=0 \mathrm{rad}$; $r_{2}=3.2 \mathrm{~mm} ; \theta_{02}=0 \mathrm{rad}$.

\section{Conclusions}

An analytical approach for solving 3D problems of material heating with laser beam was considered. The heating process was modeled using the linear nonstationary heat equation applied to cylindrical geometry. The spatial and temporal distributions of the temperature fields were considered. Using the method of variable separations and the Laplace transformation, the governing PDE with corresponding $\mathrm{BC}$ and IC was solved and the temperature field distributions were presented in closed form.

By using appropriate set of orthogonal functions, the numerical procedure was made more effective, producing saving in CPU time. The obtained numerical results improved as the number of terms used in the series of the solution increased.

Numerical examples were presented for the Al specimen with different characteristic positions of the laser beam. The convolution integral and Duhamel's principle can be used to represent arbitrary time dependence of the laser beam intensity.

For complex profiles of the laser beam the superposition principle was used. The temperature field distribution was considered and presented for two different laser beams targeting the same specimen.

The presented analytical solutions offers further advantage relative to direct monitoring since measurement of the temperature field distribution inside the 
bulk of the material is difficult to be arranged and can be usually performed in a restricted number of points.

\section{References}

[1] T. Thorslund, F.J. Kahlen, A. Kar, Temperatures, pressures and stress during laser shock processing, Optics and Lasers in Engineering 39 (2003) $51-71$

[2] S. J. Farlow, Partial Differential Equations for Scientists and Engineers, Dover Publications, INC. New York, 1993.

[3] R. M. Wood, Laser Damage in Optical Materials, Adam Hilger, Bristol and Boston, 1986.

[4] E. M. Bass, Laser Material Processing, North Holland, Amsterdam, 1983.

[5] S. Bojanic, Analyzing of laser-material interaction with condensed matter at viewpoint of physical models, $\mathrm{PhD}$ thesis, The Faculty of Electrical Engineering, University of Belgrade, Belgrade, 1997.

[6] C. A. Brebbia, J.C.F. Telles, L.C. Wrobel, Boundary Element Techniques, Springer-Verlag, Berlin, 1984.

[7] L. R. Ram-Moham, Finite Element and Boundary Element Applications in Quantum Mechanics, Oxford University Press, New York, 2002.

[8] N. Rykalin, A. Uglov, A. Kokora, Laser Machining and Welding, MIR Publishers, Moscow, 1978.

[9] R. Gospavic, M. Sreckovic, V. Popov, Modeling of laser-material interaction using semi-analytical approach, Elsevier, Mathematics and Computers in Simulations 65 (2004) 211-219.

[10] R.A. Flinn, P.K. Trojan, Engineering Materials and their Application, Houghton Mifflin Company, Boston, 1975.

[11] Y. Toyozawa, Optical Processes In Solids, Cambridge University Press, New York, 2003.

[12] E. Kreyzig, Advanced Engineering Mathematics, John Wiley \& Sons, New York, 1983.

[13] E. Jahnke, F. Emde, F. Lösch, Special functions, Nauka, Moscow, 1968.

[14] M. Abramovic, I.A. Stegun, Handbook of Mathematical Functions, Dover publications, INC., New York, 1972. 